\title{
Experiencias sobre la articulación de las funciones sustantivas en Educación Superior
}

\section{Experiences on the articulation of substantive functions in Higher Education}

\author{
Wilson Clodoveo García Guevara ${ }^{1}$, Gabriela Angelita Jara Saldaña ${ }^{1}$ y María de Lourdes Cedillo ${ }^{1}$ \\ ${ }^{1}$ Universidad Católica de Cuenca \\ *wgarciag@ucacue.edu.ec
}

DOI: https://doi.org/10.26871/killkana_social.v2i3.334

\begin{abstract}
Resumen
El presente artículo tiene como objetivo presentar las experiencias desarrolladas de los docentes de la Unidad Académica de Educación de la Universidad Católica de Cuenca en el proyecto de investigación denominado Desarrollo de la Unidad de Diagnóstico, Investigación Psicopedagógica y de Apoyo a la Inclusión, este centro brinda un servicio educativo especializado mediante la evaluación integral, asesoramiento, intervención psicopedagógica apoyando a la inclusión educativa de estudiantes que presentan necesidades educativas especiales con o sin discapacidad. Los beneficiarios fueron, estudiantes que presentan necesidades educativas especiales asociadas o no con la discapacidad de los niveles inicial, básica y bachillerato de las instituciones educativas de la provincia del Azuay junto con sus familias; los estudiantes universitarios de las carreras participantes quienes a través de un proyecto de vinculación realizaron el seguimiento y asesoramiento a las comunidades educativas en los procesos de adaptaciones curriculares. En cuanto a la metodología: se trata de una investigación cuanti-cualitativa, observacional, descriptiva, transversal y diagnóstica. Las técnicas empleadas fueron: la observación, la encuesta, técnicas sicométricas y proyectivas; los instrumentos utilizados fueron: fichas, cuestionarios, registros, entre otros, la población que intervino en la investigación está relacionada con estudiantes que presentan necesidades educativas especiales que acudieron a la Unidad; los docentes tutores y los estudiantes que realizaron las prácticas preprofesionales. Las experiencias más significativas han sido poder articular las funciones sustantivas de la Universidad como son la docencia, la investigación y la vinculación a través de este proyecto de investigación que luego se convirtió en un proyecto emblemático de vinculación con la sociedad.
\end{abstract}

Palabras clave: evaluación psicopedagógica, investigación, vinculación con la sociedad, inclusión educativa , adaptaciones curriculares.

\section{Abstract}

The present article aims to present the experiences developed by the teachers of the Academic Unit of Education of the Catholic University of Cuenca in the research project called Development of the Unit of Diagnosis, Psychopedagogical Research and Support for Inclusion, this center provides a specialized educational service through comprehensive assessment, counseling, psychopedagogical intervention supporting the educational inclusion of students who have special educational needs with or without disabilities. The beneficiaries were students with special educational needs associated or not with the disability of the initial, basic and baccalaureate levels of the educational institutions of the province of Azuay together with their families; the university students of the participating careers who through a linking project carried out the follow-up and advice to the educational communities in the processes of curricular adaptations. Regarding the methodology: it is a quantitative-qualitative, observational, descriptive, transversal and diagnostic investigation. The techniques used were: observation, survey, psychometric and projective techniques; the instruments used were: files, questionnaires, records, among others, the population that intervened in the research is related to students who have special educational needs who came to the Unit; teachers tutors and students who carried out pre-professional practices. The most significant experiences have been to articulate the substantive functions of the University such as teaching, research and linking through this research project that later became an emblematic project of links with society.

Key words: psychopedagogical evaluation, research, links with society, educational inclusion, curricular adaptations. 


\section{Introducción}

La Unidad de Diagnóstico, Investigación Psicopedagógica y de Apoyo a la Inclusión (UDIPSAI) es un centro de sicodiagnóstico que tiene como objetivo brindar un servicio educativo especializado gratuito mediante la evaluación integral, asesoramiento, intervención psicopedagógica apoyando a la inclusión educativa de estudiantes que presentan necesidades educativas especiales con o sin discapacidad. La UDIPSAI inició en noviembre del 2014 con un grupo de docentes de la Unidad Académica de Educación, cuando la Dirección de Investigación de la Universidad Católica de Cuenca convoca por segunda ocasión a "Presentación de Proyectos de Investigación",

Este proyecto inició con la aprobación y la implementación de la UDIPSAI, se ejecutó el proyecto y posteriormente se realizó la investigación de campo con la evaluación e intervención psicopedagógica a la población estudiantil que presenta necesidades educativas especiales; constituyéndose en un espacio donde el estudiante, su familia y los docentes de las instituciones educativas tenían la oportunidad de encontrar apoyo profesional para superar sus problemas en el proceso de enseñanza aprendizaje y recibir el adecuado asesoramiento y orientación familiar.

Durante la ejecución del proyecto las experiencias más significativas fueron articular las funciones sustantivas de la Universidad como son la docencia, la investigación y la vinculación; la docencia, cuando los estudiantes aplican los conocimientos teóricos, aprendidos en las aulas de clase, a través de las prácticas pre-profesionales, además la docencia está presente en las tutorías que realizan los docentes por medio de la orientación y asesoramiento a estudiantes en los procesos de evaluación sicopedagógica; la investigación se desarrolló al recolectar la información en una base de datos con las incidencias diagnósticas que permitieron a los estudiantes y docentes de la Unidad Académica de Educación realizar otros proyectos de investigación; finalmente los estudiantes de las carreras participantes a través de un proyecto de Vinculación con la Sociedad pudieron cumplir con sus horas al realizar el seguimiento de casos visitando las instituciones educativas beneficiarias. Al concluir con el periodo de ejecución, la UDIPSAI pasó a pertenecer a la Subdirección de Vinculación de la Sociedad de la Universidad Católica de Cuenca.

La UDIPSAI pretende dar cumplimento a las funciones sustantivas de educación superior contempladas en el Reglamento de Régimen Académico, 2017 en donde manifiesta que las instituciones de educación superior deberán contar con un modelo de vinculación con la sociedad, que asegure la integración de las tres funciones sustantivas de la educación superior: docencia, investigación y vinculación con la sociedad; para la gestión del conocimiento en función de sus dominios, líneas de investigación, oferta académica vigente y necesidades de la comunidad a nivel local, nacional y regional; respondiendo al principio de pertinencia. Las instituciones de educación superior podrán crear instancias institucionales específicas para gestionar la vinculación con la sociedad, a fin de generar programas, proyectos específicos o intervenciones de interés público. (Superior de Consejo de Educación, 2017)

\section{Desarrollo}

Las universidades como centros del saber cristaliza el proceso social del conocimiento: producción, difusión y aplicación de los conocimiento que no es más que la manifestación del lugar que ocupa la ciencia dentro de estas instituciones, en primer lugar, porque en las universidades se asimilan los conocimientos adquiridos por la humanidad representados en las diferentes disciplinas, en segundo lugar porque en ellas se producen nuevos conocimientos a través de las investigaciones que realizan profesores y estudiantes y en tercer lugar porque los conocimientos producidos van dimidos a transformar el entorno social. De ahí que su encargo social se concreta en tres procesos que se ponen en ejecución por acción del conocimiento: docencia, investigación y extensión. Las tres funciones; la docencia como proceso de enseñanza y aprendizaje de los conocimientos; producidos a través de la investigación científica representada en las diferentes ciencias y constituye el contenido de las disciplinas. La extensión universitaria interrelaciona la docencia y la investigación a través de la promoción de estas acciones al entorno social para satisfacer las necesidades del desarrollo cultural y la solución de problemas de la práctica social.(Fabre Batista, 2005)

La evaluación psicopedagógica consiste en una serie de procedimientos en diferentes áreas en las que el investigado presenta dificultades, dicho proceso cumple con funciones como: entrevistar, evaluar, calificar, observar, diagnosticar y recomendar.

La Evaluación Psicopedagógica es una disciplina de la Psicología que se ocupa de la medida de algunos aspectos del comportamiento e intelecto humano; esta medición se realiza por medio de metodologías básicas entre las que se encuentra las técnicas psicométricas y las proyectivas y otros métodos como la observación, la entrevista, los autoinformes, etc. (Llanezca, 2007, p.37).

Para la evaluación psicopedagógica en la UDIPSAI se implementó un equipo transdisciplinario conformado por las áreas de: psicología educativa (psicólogo educativo), psicología clínica (psicólogo clínico) y terapia del lenguaje (terapista del lenguaje/fonoaudiólogo). En cada una de las áreas los estudiantes de las diferentes carreras realizaron las prácticas pre-profesionales y los procesos de vinculación con la sociedad, con la tutoría de los docentes de las diferentes unidades académicas.

Para hacer efectiva la inclusión de los estudiantes en el sistema educativo es necesario en algunas ocasiones realizar las adaptaciones curriculares que son entendidas "como un conjunto de medidas que se toman para un determinado alumno, pero con una perspectiva sistémica, global, de centro.” (Ruiz Rodríguez, 2003, p.5) 
En la UDIPSAI, se propone incluir a los estudiantes en el sistema educativo, realizando las adaptaciones curriculares pertinentes según el caso. Existen dos tipos de adaptaciones curriculares; 1) adaptaciones de acceso al currículo, que responde a las necesidades específicas asociadas a la discapacidad, visual, auditiva, física y otras, que dificultan el acceder a las experiencias de aprendizaje, puede ser una modificación, adaptación o mejora en el sistema de comunicación docente-estudiante; estas adaptaciones conllevan un tiempo prolongando, por lo tanto disminuirá la cantidad del contenido a impartirse durante un periodo lectivo, motivo por el cual existe la posibilidad de realizar la adaptaciones a los elementos básicos del currículo, ya sea metodología, contenido, objetivos y otros, pero siempre enfocados hacia el éxito del interaprendizaje y sin perjudicar la calidad de la educación. 2) adaptaciones a los elementos básicos del currículo que hacen referencia a satisfacer las necesidades educativas de los estudiantes realizando modificaciones al plan curricular que incluye, contenidos, objetivos, metodología, recursos y sistema de evaluación (Ruiz Rodríguez, 2003, p.4)

El Documento individual de adaptación curricular (DIAC) es el documento que se elabora para especificar el tipo de adaptación que necesita un estudiante, para qué grado, en que asignaturas, las necesidades educativas especiales, apoyos técnicos externos, apoyo familiar, criterios de promoción y el seguimiento que se debe dar al caso.

Métodología.- Los participantes en este estudio fueron 2406 estudiantes que presentaron necesidades educativas especiales de las diferentes instituciones educativas de la Zona 6 de Educación remitida por los profesionales de Consejerías para evaluación sicopedagógica, docentes tutores y estudiantes que realizaron las prácticas preprofesionales y vinculación con la sociedad en la UDIPSAI. Para garantizar los aspectos éticos se presenta el consentimiento informado a los padres de familia y/o representantes legales de los estudiantes, quienes autorizaron la participación de sus representados en este estudio. Se trata de una investigación cuanti-cualitativa, observacional, transversal y diagnóstica, las técnicas empleadas fueron la encuesta, la observación, la sicometría, estudio de casos, entre otras.

Los datos fueron tabulados en base a las incidencias diagnósticas anualmente para establecer la prevalencia de las diferentes NEE, y; a partir de ello se obtuvieron las conclusiones en donde se puedo constatar que la población atendida corresponde en un porcentaje mayor al sector público y la aceptación es satisfactoria por parte de los docentes y directivos de las instituciones educativas, lo cual servirá de sustento para planificar actividades de prevención y seguimiento.

El objetivo general que se planteó en la investigación fue determinar la articulación de las funciones sustantivas de la universidad en el proceso de Evaluación Psicopedagógica en la UDIPSAI.

\section{Resultados}

Durante los 45 meses de ejecución se atendieron a 2406 estudiantes con necesidades educativas especiales asociadas o no a la discapacidad pertenecientes a instituciones educativas de la provincia del Azuay, el $65 \%$ pertenecen al género masculino, y el $35 \%$ al femenino.

De las instituciones educativas atendidas el $54 \%$ pertenecen al sector fiscal, el $45 \%$ al sector particular y el $1 \%$ al sector fiscomisional; referente a la atención en las diferentes áreas se pudo constatar que área de mayor incidencia en cuanto a la evaluación, fue la de Sicología Educativa con el $56 \%$ seguida de Sicología Clínica con el $34 \%$ y Terapia de Lenguaje el $10 \%$.

En el área de Sicología Educativa se identificó a los estudiantes que pertenecen a inclusión educativa y para los cuales se elaboró el Documento Individual de Adaptación Curricular (DIAC) en donde se especificó las adaptaciones curriculares, esta población representó el $26 \%$.

La UDIPSAI nace como un proyecto de investigación, a partir del cual se elaboró un proyecto de vinculación con la sociedad para que los estudiantes de la carrera de sicología educativa pongan en práctica la teoría aprendida en las aulas de clase, hasta la fecha han participado 45 docentes tutores de las diferentes carreras que oferta la Universidad Católica de Cuenca: 19 de la carrera de Psicología educativa y 4 de Psicología Clínica y 2 de Trabajo Social 1 de las TCs, 2 de Fonoaudiología, 16 de Odontología, 1 de Comunicación.

Los estudiantes que realizaron Vinculación con la sociedad y prácticas pre-profesionales fueron: 83 de la carrea de Sicología Educativa, 19 de Psicología Clínica, 53 de 0domtología, 16 de las TCs, 8 de Trabajo Socia, 3 de comunicación, realizaron las prácticas con quienes se genera el presente estudio.

El proceso de evaluación psicopedagógica, atención e intervención con los estudiantes que presentan necesidades educativas especiales asociadas o no a la discapacidad, la elaboración de las adaptaciones curriculares para hacer efectiva la inclusión educativa, motivó los docentes y estudiantes a investigar, y comunicar los resultados a través de elaboración de artículos académicos y participación en Congresos nacionales e internacionales con ponencias orales tales como: artículo científico con el tema "Barreras para el acceso a la Universidad de las personas con discapacidad en Ecuador"; "La discapacidad intelectual y el aprendizaje de la lectoescritura" y "Prevalencia de los factores etiológicas que influyen en los problemas de aprendizaje" utilizando la información de la base de datos de los resultados de los diagnósticos sicopedagógicos. Además, se participó con ponencias en el II Congreso Internacional de Ciencias Pedagógicas en Guayaquil en abril del 2016 con los temas "Desarrollo de la Unidad De Diagnóstico, Investigación Psicopedagógica y de Apoyo a la Inclusión (UDIPSAI-UCACUE) para la atención de estudiantes con necesidades educativas especiales asociadas o no a la discapacidad" y "La discapacidad intelectual y el aprendi- 
zaje de la lectoescritura en estudiantes que asisten a la UDIPSAI-UCACUE"; en el IX Encuentro Internacional de estudiantes de Sicología en la Universidad de la HabanaCuba en el mes de octubre de 2016 con el tema: "La evaluación psicopedagógica y la inclusión educativa", "La Unidad de Diagnóstico, Investigación Psicopedagógica y de Apoyo a la Inclusión como espacio de prácticas pre profesionales y vinculación con la sociedad" y "Avances que se han logrado en la Unidad de Diagnóstico, Investigación Psicopedagógica y de Apoyo a la Inclusión"; en el III Congreso Internacional Educación y Universidad para la Transformación Social organizado por la UNAE en mayo de 2018 con los temas "Necesidades educativas especiales en educación superior", "La discapacidad intelectual abordada desde la perspectiva psicopedagógica", "Prácticas de evaluación psicopedagógica como apoyo a la inclusión"; en el IV Congreso Internacional de Ciencias Pedagógicas con los temas "Docencia, investigación y vinculación con la sociedad y la evaluación psicopedagógica" y "Discapacidad multireto: evaluación, diagnóstico, intervención e inclusión educativa..”; en el IX Congreso Internacional de Psicología y Educación en España junio de 2018 con el tema "Propuesta de programa de educación inclusiva en la Universidad Católica de Cuenca para estudiantes que presentan necesidades educativas especiales asociadas o no a la discapacidad" y en las Jornadas Repensando la Educación Superior en Ecuador, América Latina y el caribe: a cien años de la Reforma Universitaria de Córdoba en Quito junio 2018 con los temas “ Inclusión Educativa multireto en la Universidad Católica de Cuenca. Un estudio de caso" y "Propuesta de educación inclusiva en educación superior"; finalmente se publica el artículo académico en revista Killkanas. con el tema: "Prácticas inclusivas de los docentes".

Los estudiantes de la carrera de Sicología Educativa realizaron la investigación sobre el nivel de impacto proceso que consiste en aplicar encuestas a los directivos de las instituciones educativas beneficiarias para determinar el nivel de satisfacción del servicio brindado por la UDIPSAI. De las encuestas aplicadas en julio del 2015 para el nivel impacto; el $90 \%$ de directivos manifiestan que el servicio que presta la UDIPSAI permite al docente mejorar el proceso de enseñanza- aprendizaje de los estudiantes con NEE y el $10 \%$ afirma lo contrario.

En el proceso de recategorización de la Universidad Católica de Cuenca en el mes de octubre del 2016, se socializó los avances de la UIDPSAI, estadísticas de la atención brinda, de estudiantes y docentes participantes en la presentación de proyectos de investigación y vinculación de la Universidad ante el Consejo de Evaluación, Acreditación y Aseguramiento de la Calidad de la Educación Superior (CEAACES).

\subsection{Conclusión}

De la encuesta aplicada a los docentes y directivos de las instituciones educativas se concluye que el servicio brin- dado por la UDIPSAI beneficia a la comunidad educativa. (autoridades, docentes, estudiantes, padres y/o representantes), la evaluación sicopedagógica que presta la UDIPSAI satisface las demandas de los estudiantes con necesidades educativas especiales, los docentes cumplen con las estrategias metodológicas establecidas en el Desarrollo de la Unidad de Diagnóstico, Investigación Psicopedagógica y de Apoyo a la Inclusión para la atención de estudiantes con necesidades educativas especiales asociadas o no a la discapacidad informes sicopedagógicos y documento individual de adaptación curricular.

Luego de tabulada las encuestas de seguimiento realizadas por los estudiantes de la Universidad Católica de Cuenca a los docentes de las instituciones educativas beneficiarias se concluye que la evaluación sicopedagógica y las adaptaciones curriculares permitieron mejorar las condiciones de inclusión de los estudiantes con necesidades educativas especiales.

Dentro del proceso de vinculación con la sociedad los estudiantes de la carrera de Sicología Educativa narran sus experiencias positivas de la práctica profesional vivida en el proyecto UDIPSAI, destacando el apoyo recibido de parte de los docentes tutores, de coordinanación y de la parte administrativa. Una estudiante en lo que se refiere al proceso de evaluación sicopedagógica manifestó que atendió a varios niños, niñas y adolescentes que presentaban necesidades educativas especiales como hiperactividad, déficit de atención, discapacidad intelectual y otros; cada estudiante evaluado le permitió vivir experiencias significativas, poniendo en práctica la parte teórica aprendida en las aulas de clase.

Los docentes y estudiantes realizaron proyectos de investigación bajo la modalidad de titulación, utilizando las experiencias en los procesos de evaluación psicopedagógica y la información filtrada en la base de datos. Además, se elaboró artículos académicos y se participó en ponencias a nivel nacional e internacional.

El proyecto de investigación inició en noviembre del 2014 y estaba presupuestado por 2 años por lo que concluyó en octubre del 2016; debido a la alta demanda de los beneficiarios, los resultados del nivel de impacto y las cartas de satisfacción de las instituciones educativas se elaboró un propuesta para dar continuidad al proyecto UDIPSAIUCACUE, elaborado desde la Unidad Académica de Educación, convirtiéndose en un proyecto de vinculación con la sociedad, dando así cumplimiento a las recomendaciones dadas por el Reglamento de Régimen Académico, respecto a las funciones sustantivas de la Universidad, en las que todo proyecto de investigación debe concluir con una propuesta de vinculación y viceversa.

Dentro de las experiencias más significativas para los que participaron en el proyecto de investigación, ha sido articular las funciones sustantivas de la Universidad como son la docencia, a través de tutorías, orientación y asesoramiento (proceso de inducción, guía y acompañamiento), la investigación y la vinculación a través de este proyecto 
de investigación que luego se convirtió en un proyecto emblemático de vinculación con la sociedad de la Universidad Católica de Cuenca.

\section{Referencias Bibliográficas}

Fabre Batista, G. C. (2005). SEDICI. Descargado de http://sedici.unlp.edu.ar/ bitstream/handle/10915/24694/ Documento_completo.pdf?sequence= $1 \&$ isAllowed $=y$

Llanezca, D. F. M. G. (2007). Instrumentos de Evaluación psicológica. La Habana: Ciencias Médicas.

Ruiz Rodríguez, E. (2003). Adaptaciones curriculares individuales para los alumnos con síndrome de Down. Revista Sindrome de Down, 4-15.

Superior de Consejo de Educación. (2017). CEE Consejo de Educación Superior. Descargado de http:// www.ces.gob.ec/lotaip/2018/Enero/
AnexosProcu/An-lit-a2-Reglamento\%

20 de20Régimen\%20Académico.pdf

Aceptado: 3 de septiembre de 2018 
